Received: 2015.02.14 Accepted: 2015.07.01 Published: 2015.10.08

Authors' Contribution: Study Design A Data Collection B Statistical Analysis C Data Interpretation D Manuscript Preparation E Literature Search F Funds Collection G

Corresponding Author: Conflict of interest:

E 1 Mohammed Al-Sofiani

E 2 Peterkin Lee Kwen

None declared
1 Department of Medicine, South Buffalo Mercy Hospital, University at Buffalo, Buffalo, NY, U.S.A.

2 Department of Neurology, South Buffalo Mercy Hospital, University at Buffalo, Buffalo, NY, U.S.A.

Mohammed Al-Sofiani, e-mail: Dr_moh2008@hotmail.com

Patient:

Male, 83

Final Diagnosis:

Symptoms:

Midbrain infarction

Medication:

Diplopia

Clinical Procedure:

Specialty:

Computed tomography of the head and magnetic resonance image of the brain

Neurology

Objective:

\title{
Rare disease
}

Background:

Diplopia is a common subjective complaint that can be the first manifestation of a serious pathology. Here, we report a rare case of midbrain infarction involving the lateral subnucleus of the oculomotor nuclear complex presenting as diplopia, with no other stroke manifestations.

Case Report: An 83-year-old right-handed white man with past medical history of diabetes mellitus, hypertension, dyslipidemia, and coronary artery disease presented to the emergency department (ED) with diplopia and unsteadiness. Two days prior to admission, the patient woke up with constant horizontal diplopia and unsteadiness, which limited his daily activities and led to a fall at home. He denied any weakness, clumsiness, nausea, vomiting, photophobia, fever, or chills. Ocular exam showed a disconjugate gaze at rest, weakness of the left medial rectus muscle, impaired convergence test, and bilateral 3-mm reactive pupils. The diplopia resolved by closing either eye. The remaining extraocular muscles and other cranial nerves were normal. There was no nystagmus, ptosis, or visual field deficit. Sensation, muscle tone, and strength were normal in all extremities. Magnetic resonance imaging (MRI) of the brain revealed a tiny focus of restricted diffusion in the left posterior lateral midbrain.

Conclusions: A thorough history and physical examination is essential to diagnose and manage diplopia. Isolated extraocular palsy is usually thought to be caused by orbital lesions or muscular diseases. Here, we report a case of midbrain infarction manifested as isolated medial rectus palsy.

MeSH Keywords: $\quad$ Diplopia $\bullet$ Oculomotor Nerve • Stroke

Full-text PDF: http://www.amjcaserep.com/abstract/index/idArt/893875

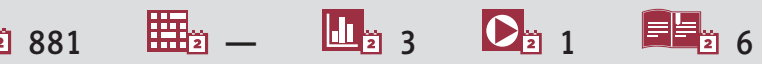




\section{Background}

Diplopia is a common subjective complaint that can be the first manifestation of a serious pathology. Isolated medial rectus palsy as the presenting manifestation of midbrain infarction is rare, particularly when no other stroke manifestations are identified. Here, we report a case of midbrain infarction that presented as isolated medial rectus palsy, and outline the clinical approach taken to identifying the etiology of diplopia in this patient.

\section{Case Report}

An 83-year-old right-handed white man presented to the ED with diplopia and unsteadiness for 2 days. Two days prior to admission, the patient woke up with constant horizontal diplopia, where single objects appeared as double (side-by-side images). He also had unsteadiness, which limited his daily activities and led to a fall at home. He denied any weakness or clumsiness, numbness or tingling, slurred speech, nausea, vomiting, photophobia, fever, or chills.

His past medical history was significant for diabetes mellitus, hypertension, dyslipidemia, and coronary artery disease. He denied smoking, drinking alcohol, or using illicit drugs.

On physical exam, he was afebrile, with respiratory rate of 16 breaths/min, blood pressure of $170 / 80 \mathrm{mmHg}$, heart rate of $60 \mathrm{bpm}$, and oxygen saturation of $98 \%$ on room air. An ocular exam showed a disconjugate gaze at rest, weakness of the left medial rectus muscle, impaired convergence test, and bilateral 3-mm reactive pupils (Figure 1; Video 1 ). The diplopia resolved by closing either eye. The remaining 5 extraocular muscles (superior rectus, inferior rectus, lateral rectus, superior oblique, and inferior oblique) and other cranial nerves were normal. There was no nystagmus, ptosis, or visual field deficit. Sensation, muscle tone, and strength were normal in all extremities.

At this point, our differential diagnoses were ischemic stroke involving the lateral subnuclei of the oculomotor nucleus, posterior communicating artery aneurysm, cavernous sinus aneurysm, intranuclear ophthalmoplegia (INO), refractive error, or idiopathic oculomotor nerve palsy.

A CT scan of the head showed a small old left putamen lacunar infarct. MRI of the brain revealed a tiny focus of restricted diffusion in the left posterior lateral midbrain and findings suggestive of advanced amyloid angiopathy (Figures 2, 3).

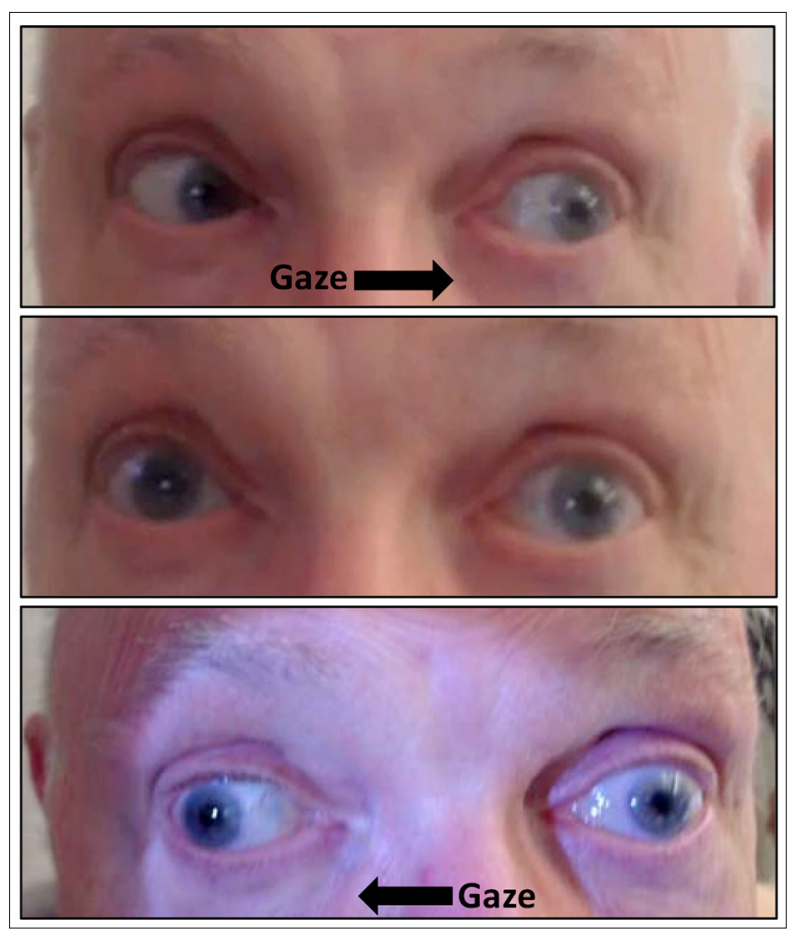

Figure 1. Right gaze weakness affecting the left eye.

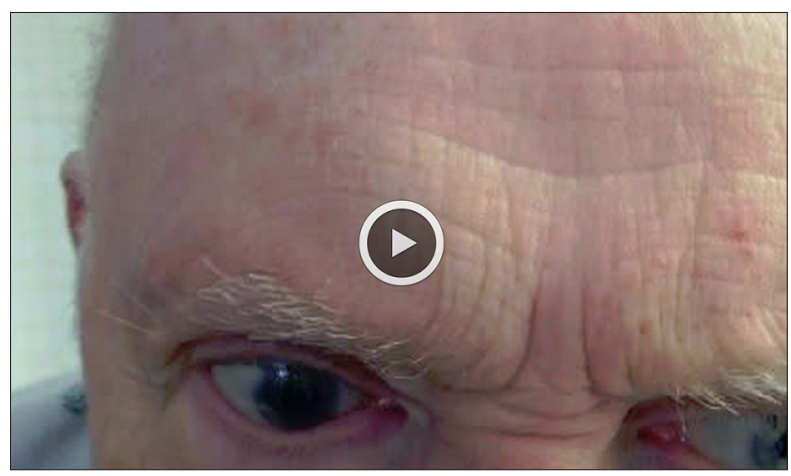

Video 1. Examination of the extraocular muscle function showing impaired adduction of the left eye.

The patient was started on aspirin $81 \mathrm{mg} /$ day and was advised to avoid other anti-platelet agents or anti-coagulants due to the high risk of bleeding from cerebral amyloid angiopathy.

\section{Discussion}

Diplopia is a common subjective complaint encountered by health care providers. Identifying the etiology of diplopia can be a challenge because of the long list of differential diagnoses. Thus, a thorough history and physical examination is essential to diagnose and manage diplopia. Our patient had binocular diplopia, defined as "diplopia (that) resolves when the affected eye is occluded", which indicates misalignment of the visual axes as being the cause of the diplopia. In contrast, 


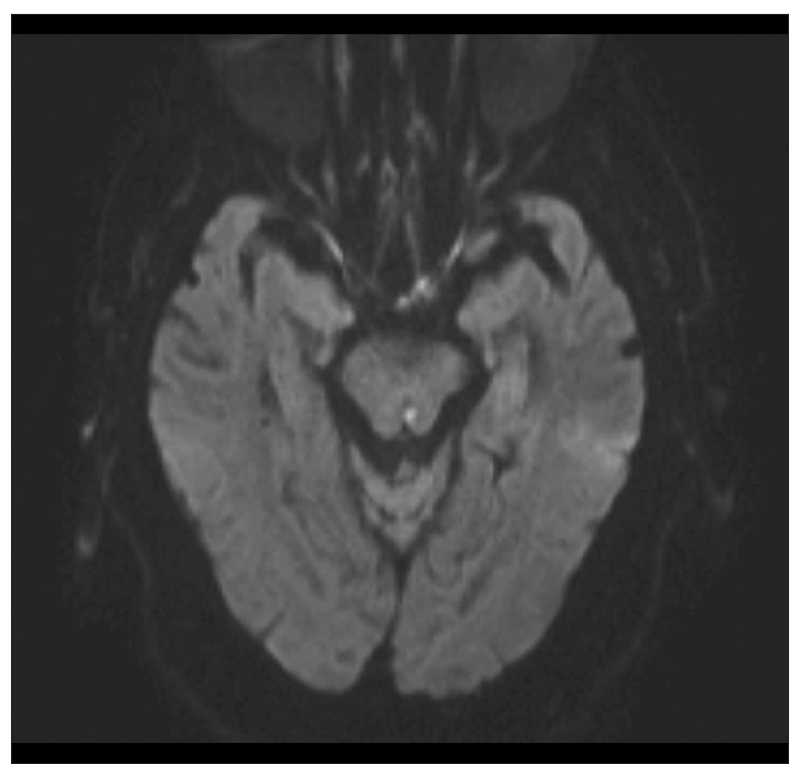

Figure 2. MRI of the brain showing a tiny focus of restricted diffusion in the left posterior lateral midbrain.

monocular diplopia, defined as "diplopia (that) persists when the affected eye is occluded" is usually caused by ophthalmological pathology, such as refractive error [1]. The binocular diplopia in our patient limited the differential diagnosis to impaired neural control or function of the extraocular muscles. Horizontal diplopia further shortened the differential diagnosis list to impairment of the medial rectus, lateral rectus, or both. The restricted adduction of the left eye (Video 1) suggests dysfunction of the left medial rectus muscle, which is one of the extraocular muscles supplied by the lateral subnuclei of the oculomotor nuclear complex (CNIII). The lateral subnucleus of the oculomotor nuclear complex is composed of 3 subnuclei, which, from dorsal to ventral, supply the inferior rectus, inferior oblique, and medial rectus muscles, respectively [2]. A typical presentation of complete oculomotor nerve palsy includes "down and out" position of the affected eye, ipsilateral ptosis, and fixed dilated pupil [3)] However, partial oculomotor nerve palsy is more commonly encountered in clinical practice than is complete oculomotor nerve palsy. Pupil-sparing oculomotor nerve palsy is usually caused by vascular microinfarction, but this is true only when there is a complete ptosis of the eye [1]. The complete pupil-sparing in our patient made a compressive lesion, such as posterior communicating artery aneurysm, less likely. Another differential diagnosis in our case was INO. However, the absence of nystagmus in the contralateral abducting eye and the impaired convergence test in our patient favored the diagnosis of isolated medial rectus palsy over INO [1]. In addition to the physical exam findings, the advanced age of our patient and history of hypertension,

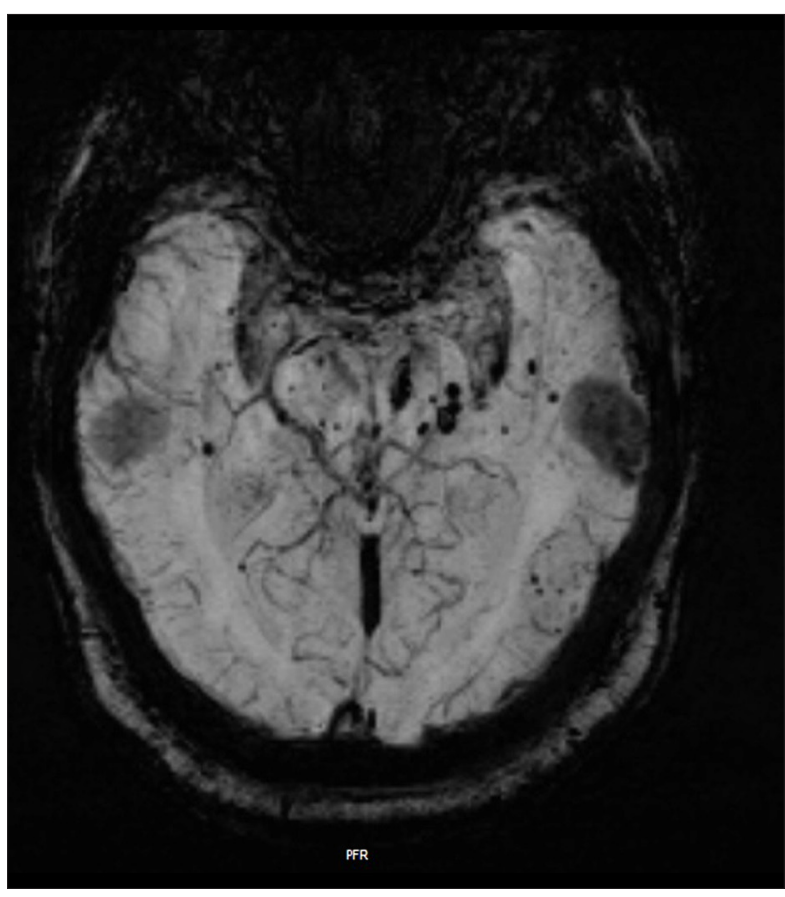

Figure 3. MRI of the brain showing findings suggestive of advanced amyloid angiopathy.

diabetes, dyslipidemia, and coronary artery disease supported the diagnosis of midbrain infarction involving the lateral subnuclei of the oculomotor nuclear complex. This diagnosis was confirmed by the MRI, which also revealed an incidental finding of advanced amyloid angiopathy (Figures 2, 3).

Isolated unilateral extraocular palsy is usually thought to be caused by orbital lesions or muscular diseases. In this report, we presented a rare case of midbrain infarction involving the lateral subnuclei of the occulomotor nuclear complex presenting as isolated medial rectus palsy. Isolated superior and inferior rectus palsy have been reported after midbrain infarction $[4,5]$. In fact, a similar case of left medial rectus palsy, but with left partial ptosis, was reported by Rabadi as a consequence of midbrain infarction [6].

\section{Conclusions}

Unilateral isolated medial rectus nuclear palsy can be the only manifestation of midbrain infarction. Systematic clinical approach with appropriate history and physical examination is essential to elucidate the etiology of diplopia and to avoid missing a serious underlying diagnosis, such as cerebrovascular accidents. 


\section{References:}

1. Danchaivijitr C, Kennard C: Diplopia and eye movement disorders. J Neurol Neurosurg Psychiatry, 2004; 75: iv24-31

2. Wilson-Pauwels L, Akesson EJ, Stewart PA, Spacey SD: Cranial Nerves in Health and Disease. $2^{\text {nd }}$ ed. London: BC Decker; 2002

3. Bruce BB, Biousse V, Newman NJ: Third nerve palsies. Semin Neurol, 2007; 27(3): 257-68
4. Kwon JH, Kwon SU, Ahn HS et al: Isolated superior rectus palsy due to contralateral midbrain infarction. Arch Neurol, 2003; 60(11): 1633-35

5. Takano M, Aoki K: [Midbrain infarction presenting isolated inferior rectus nuclear palsy.] Rinsho Shinkeigaku, 2000; 40(8): 832-35 [in Japanese]

6. Rabadi MH, Beltmann MA: Midbrain infarction presenting isolated medial rectus nuclear palsy. Am J Med, 2005; 118(8): 836-37 